\title{
A STUDY OF THE MODIFIED GINZBURG-LANDAU TYPE EQUATION FOR A JOSEPHSON JUNCTION
}

\author{
T. L. Boyadjiev ${ }^{1}$, Z. D. Genchev ${ }^{2}$ \\ ${ }^{1}$ Faculty of Mathematics and Computer Science, \\ University of Sofia "St. Kl. Ohridski", BG-1164, Sofia, Bulgaria \\ ${ }^{2}$ Institute of Electronics, Bulgarian Academy of Sciences, BG-1784, Sofia, Bulgaria
}

(Received October 1, 2001)

\begin{abstract}
The formalism of Ginzburg-Landau (GL) provides a simple method to study the global properties of non-homogeneous superconducting structures. In this paper we investigate a class of supercondicting/normal/superconducting (SNS) structures (sandwiches) with plane boundaries on the basis of the modified Ginzburg-Landau (GL) type equations. The corresponding non-linear boundary value problem for the amplitude of the order parameter is solved numerically. We show, that for fixed values of the phenomenological coefficients of the SNS structure there exist various solutions with different energies and their own phase differences. The two basic solutions with minimal energy in the case of an infinite sandwich are also obtained analytically. The resulted current density-phase offset dependence is constructed. Due to the existence of different nonlinear terms in the normal and superconducting regions, this dependence is not sinusoidal. In order to estimate the influence of the phenomenological coefficients on the form of current density - phase offset curve - a Fourier decomposition of this curve is also made.

Key words: Modified Ginzburg-Landau equation, Josephson junction, supercurrent-phase relation, nonlinear boundary value problem, bifurcation, Fourier coefficients.

PACS number(s): 74.20.De, 02.60.Lj, 02.70.Dh.
\end{abstract}

\section{INTRODUCTION}

The physics of Josephson junctions is based on an usual sinusoidal supercurrent-phase difference relation

$$
j_{s}(\Delta \phi)=j_{c} \sin \Delta \phi
$$

After the discovery of the Josephson effect, it became clear that, apart from an insulating tunnel structure, any sufficiently short localized weak link such as a very short constriction in the cross-section of a superconductor, a point contact between two superconductors, as well as two superconductors separated by a thin layer of normal metal, could be used as a Josephson junction, obeying the current-phase relations, usually different from (1). This fact forced Licharev [1] and Waldram [2] to propose a generalized definition: a weak link is supposed to show a Josephson behaviour if the supercurrent-phase relation is a single-valued and odd analytical function which can be represented as a Fourier series

$$
j_{s}(\Delta \phi)=\sum_{n=1}^{\infty} a_{n} \sin (n \Delta \phi)
$$

The crossover between an ideal Josephson behaviour and an uniform superconducting flow was studied by solving exactly the usual Ginzburg-Landau (GL) equation for a 1-D superconductor in the presence of an effective $\delta$-function potential of arbitrary strength (see, for example [3]). Recently, a modified GL type model has been formulated [4]. This model could equally well be applied to a boundary between different superconductors, superconductor-insulator, and superconductornormal metal. The purpose of our paper is to apply this modified GL model for calculating the supercurrentphase relation and the crossover between Josephson behaviour and uniform superconducting flow.

We would like to mention that the SNS structures are discussed in recent references $[5-7]$.

\section{FORMULATION OF THE PROBLEM}

We focus our attention only on the current-conserving solutions of the modified GL equations [4], in which a nonzero current across the boundary is associated with a linearly varying asymptotic phase (see below the necessary boundary conditions). Let us accept an onedimensional approximation (a dependence of all relevant quantities is only on the coordinate $x$ across the boundary). Then the magnetic field of our uniform current density will depend at least on one of the transverse coordinates and in one-dimensional approximation this field could be neglected. Thus, the order parameter $\widetilde{\psi}(x)$ satisfies the following equations:

$$
-\frac{\hbar^{2}}{2 m_{s}} \widetilde{\psi}^{\prime \prime}+a_{s} \tilde{\psi}+b_{s}|\widetilde{\psi}|^{2} \widetilde{\psi}=0
$$




$$
j_{s}=-\frac{i \hbar e_{s}}{2 m_{s}}\left[\widetilde{\psi}^{*} \widetilde{\psi}^{\prime}-\tilde{\psi} \widetilde{\psi}^{* \prime}\right]
$$

for the superconducting domain $x \in(-\infty,-\tilde{d} / 2) \cup$ $(\tilde{d} / 2, \infty)$, and

$$
\begin{aligned}
& -\frac{\hbar^{2}}{2 m_{n}} \widetilde{\psi}^{\prime \prime}+a_{n} \tilde{\psi}+b_{n}|\widetilde{\psi}|^{2} \tilde{\psi}=0, \\
& j_{n}=-\frac{i \hbar e_{n}}{2 m_{n}}\left[\widetilde{\psi}^{*} \widetilde{\psi}^{\prime}-\tilde{\psi} \widetilde{\psi}^{* \prime}\right],
\end{aligned}
$$

for the normal domain $x \in(-\tilde{d} / 2, \tilde{d} / 2)$. The conditions at the N-S interfaces $x= \pm \tilde{d} / 2$ are

$$
[\widetilde{\psi}]=0, \quad\left[\frac{m_{s}}{m_{n}} \frac{d \tilde{\psi}}{d x}\right]=0
$$

so that $j_{s}=j_{n}=j$. From now on $a_{s}, a_{n}, b_{s}$, and $b_{n}$ are given GL phenomenological coefficients, and $[f]$ denotes [4] a jump of the enclosed function $f(x)$ across the points $x= \pm \tilde{d} / 2$. The quantities $e_{s}$ and $e_{n}$ are equal to the charge of the superconducting charge carriers, and in the following are both equal to twice the electron charge. We are free to choose the value of one of the effective masses $m_{s}$ and $m_{n}$. Usually $m_{s}$ (the effective mass of superconducting electrons) is chosen to be twice the electron mass. This leaves the mass $m_{n}$ as a parameter depending on the normal material.

We suppose that $a_{s}=-\left|a_{s}\right|, a_{n}>0, b_{s}>0, b_{n} \geq 0$ and define the coherence length $\xi=\hbar / \sqrt{2 m_{s}\left|a_{s}\right|}$, as well as the dimensionless distances $z=x / \xi, d=\tilde{d} / \xi$, the order parameter $\psi(z)=\tilde{\psi}(z \xi) \sqrt{b_{s} /\left|a_{s}\right|}$, and the current density

$$
J=\sqrt{\frac{m_{s}}{2\left|a_{s}\right|}} \frac{b_{s}}{\left|a_{s}\right| e_{s}} j .
$$

In order to make a further comparison with other papers we introduce an equivalent representation of Eq. (5) $J=2 \pi \xi \Lambda j / \phi_{0}$, where $\Lambda=m_{s} / n_{0} e_{s}^{2}=\mu_{0} \lambda_{L}^{2}$, and $n_{0}=\sqrt{\left|a_{s}\right| / b_{s}}$ is the equilibrium concentration of superelectrons, $\lambda_{L}$ is the well-known London penetration depth, $\phi_{0}=h / 2 e=h / e_{s}$ is the magnetic flux quantum.

With the definitions given above our problem is stated as follows

$$
\begin{aligned}
& \psi^{\prime \prime}+\left(1-|\psi|^{2}\right) \psi=0, \\
& (-\infty<z<-d / 2) \cup(d / 2<z<\infty), \\
& J=-\frac{i}{2}\left[\psi^{*} \psi^{\prime}-\psi \psi^{* \prime}\right]
\end{aligned}
$$

and

$$
\begin{aligned}
& \frac{m_{s}}{m_{n}} \psi^{\prime \prime}-\frac{a_{n}}{\left|a_{s}\right|} \psi-\frac{b_{n}}{b_{s}}|\psi|^{2} \psi=0, \quad|z|<d / 2, \\
& J=-\frac{i}{2} \frac{m_{s}}{m_{n}}\left[\psi^{*} \psi^{\prime}-\psi \psi^{* \prime}\right] .
\end{aligned}
$$

\section{ANALYTICAL SOLUTION FOR A THIN NORMAL LAYER}

Let us introduce the parameters

$$
m=\frac{m_{n}}{m_{s}}, \alpha=\frac{m_{n} a_{n}}{m_{s}\left|a_{s}\right|} \equiv m \frac{a_{n}}{\left|a_{s}\right|}, \beta=\frac{m_{n} b_{n}}{m_{s} b_{s}} \equiv m \frac{b_{n}}{b_{s}} .
$$

The case $\alpha=-1, \beta=m=1$ corresponds to an uniform superconductor occupying the whole space $(-\infty, \infty)$.

In both Eqs. (6) and (7) we set $\psi(z)=R(z) \exp [i \varphi(z)]$ and find

$$
\begin{aligned}
& R^{\prime \prime}+R-R^{3}-\frac{J^{2}}{R^{3}}=0, \quad|z|>d / 2, \\
& R^{\prime \prime}-\alpha R-\beta R^{3}-m^{2} \frac{J^{2}}{R^{3}}=0, \quad|z|<d / 2 ; \\
& R^{2} \varphi^{\prime}= \begin{cases}J, & |z|>d / 2, \\
m J,|z|<d / 2 ;\end{cases}
\end{aligned}
$$

Let us introduce the function

$$
\begin{aligned}
\delta(z ; 1-c) & =1+(1-c)\left[H\left(z-\frac{d}{2}\right)\right. \\
& \left.-H\left(z+\frac{d}{2}\right)\right]= \begin{cases}1, & |z|>d / 2 \\
c, & |z|<d / 2\end{cases}
\end{aligned}
$$

$H(z)$ is the Heaviside function. Then Eqs. (8), (9) can be written as

$$
\begin{aligned}
& R^{\prime \prime}+\delta(z ; 1+\alpha) R-\delta(z ; 1-\beta) R^{3} \\
& -\delta\left(z ; 1-m^{2}\right) \frac{J^{2}}{R^{3}}=0 .
\end{aligned}
$$

If the thickness $d \rightarrow+0$, we have $H(z-d / 2)-$ $H(z+d / 2) \approx-d \delta(z)$, so that the case of small $d$ can be formulated as follows

$$
\begin{aligned}
& R^{\prime \prime}+\left[1-g_{1} \delta(z)\right] R-\left[1-g_{2} \delta(z)\right] R^{3} \\
& -\left[1-g_{3} \delta(z)\right] \frac{J^{2}}{R^{3}}=0 .
\end{aligned}
$$

Here we merely substitute 


\section{T. L. BOYADJIEV, Z. D. GENCHEV}

$$
\begin{aligned}
& g_{1} \equiv(1+\alpha) d=d\left(1+m \frac{a_{n}}{\left|a_{s}\right|}\right), \\
& g_{2} \equiv(1-\beta) d=d\left(1-m \frac{b_{n}}{b_{s}}\right) \\
& g_{3} \equiv\left(1-m^{2}\right) d .
\end{aligned}
$$

It is clear, that for very thin normal layers, when $d \rightarrow+0$, the parameters $g_{i},(i=1,2,3)$, scaling like $d$ should be also small. This limiting case corresponds to uniform superconducting flow and to small deviations from this state. However, if the value of $d$ is fixed for a relatively thin layer (for example, $d=0.2$ in our numerical study) the situation is quite different. In this case the strength of the $\delta$-function potentials in Eq. (12) can be quite large. Moreover, for normal materials with very low electrical conductivity the effective mass $m_{n}$ can take very large values as compared to $m_{s}$, so that we can have $m \rightarrow \infty$. Thus, for relatively thin layer generally we have

$$
g_{1} \in[0, \infty), \quad g_{2} \in(-\infty, d], \quad g_{3} \in(-\infty, 0] .
$$

Under this assumption we can achieve our goal to investigate the full crossover from the Josephson effect to that of bulk superconducting flow.

A class of monotonic at $z>0(z<0)$ solutions of Eq. (12) are found to be

$$
R^{2}(z)=a+b \tanh ^{2}\left[u\left(|z|+z_{0}\right)\right],
$$

where $a(2-a)^{2}=8 J^{2}, 0 \leq a \leq 2 / 3, b=1-3 a / 2$, $u=\sqrt{b / 2}, b=a B^{2}$, and the quantity $y_{0} \equiv \tanh \left(u z_{0}\right)$ satisfies the condition $\left(0 \leq y_{0} \leq 1\right)$

$$
\begin{aligned}
& \sqrt{2 b} B^{2} y_{0}\left(1-y_{0}^{2}\right)=g_{1}\left(1+B^{2} y_{0}^{2}\right) \\
& -a g_{2}\left(1+B^{2} y_{0}^{2}\right)^{2}-\frac{g_{3} J^{2}}{a^{2}\left(1+B^{2} y_{0}^{2}\right)} .
\end{aligned}
$$

If $g_{2}=0$ and $g_{3}=0$ we recover Eq. (15) from [3].

In the limit $d \rightarrow+0$ Eq. (14) has two solutions: $y_{0}=A_{0} d$ and $y_{0}=1-C_{0} d / 2$, where the first order approximations for quantities $A_{0}>0$ and $C_{0}>0$ are given by the following formulae

$$
\begin{aligned}
A_{0} & =(2 b)^{-1 / 2} B^{-2}\left[1+\alpha-a(1-\beta)-\frac{\left(1-m^{2}\right) J^{2}}{a^{2}}\right], \\
C_{0} & =(2 b)^{-1 / 2} B^{-2}\left[(1+\alpha)\left(1+B^{2}\right)\right. \\
& \left.-a(1-\beta)\left(1+B^{2}\right)^{2}-\frac{\left(1-m^{2}\right) J^{2}}{a^{2}\left(1+B^{2}\right)}\right] .
\end{aligned}
$$

The existence of two solutions is also confirmed by our numerical results given in Sec. 4.

Now we will introduce the phase offset $\Delta \varphi$. The first integral (10) can be rewritten as $R^{2}(z) \varphi^{\prime}(z)=\delta(z ; 1-$ $m$ ) $J$, so that for small $d$ we have

$$
R^{2}(z) \varphi^{\prime}(z)=[1-d(1-m) \delta(z)] J .
$$

Due to the fact that the boundary conditions for the order parameter at $z \rightarrow \pm \infty$ must be

$$
R^{\prime}( \pm \infty)=0, \quad \varphi(z)=\frac{J}{R_{\infty}^{2}} z \pm \frac{\Delta \varphi}{2}, z \rightarrow \pm \infty,
$$

we derive from Eq. (15)

$$
\Delta \varphi=-\frac{J d(1-m)}{R^{2}(0)}+J \int_{-\infty}^{\infty}\left(\frac{1}{R^{2}(z)}-\frac{1}{R_{\infty}^{2}}\right) d z .
$$

Here $R_{\infty}=R( \pm \infty)>0$ and $R^{2}(0)=a+b y_{0}^{2}=$ $a\left(1+B^{2} y_{0}^{2}\right)$. Then, by calculating the integral in the above equation, we finally find

$$
\begin{aligned}
\Delta \varphi & =2\left\{\arctan B-\arctan \left(B y_{0}\right)\right\} \\
& -\frac{d J(1-m)}{a\left(1+B^{2} y_{0}^{2}\right)} .
\end{aligned}
$$

If the ratio $m=1$, this result formally coincides with Eq. (16) in [3].

Let us consider the special case of small current $J \rightarrow$ +0 . Then, from the usual sinusoidal Josephson relation (1) in a linear approximation we get

$$
\Delta \varphi=\arcsin \left(J / j_{c}\right) \simeq J / j_{c} .
$$

Eq. (14) is simplified considerably if $J=0$ and reduces to the following equation

$$
\sqrt{2}\left(1-Y_{0}^{2}\right)=g_{1} Y_{0}-g_{2} Y_{0}^{3},
$$

where $Y_{0}=y_{0}(J=0)$. For small $J$ we have $b \simeq 1, q=$ $2 J^{2}, B=1 /(\sqrt{2} J), 1 \gg B^{-1}$ and the right-hand-side of Eq. (16) can be replaced by a term proportional to $J$ :

$$
\Delta \varphi=2 J\left[\sqrt{2}\left(\frac{1}{Y_{0}}-1\right)-\frac{d(1-m)}{2 Y_{0}^{2}}\right] .
$$

By coupling Eqs. (17) and (19) we derive an approximate estimation for the critical current

$$
\frac{1}{j_{c}}=2 \sqrt{2}\left(\frac{1}{Y_{0}}-1\right)-\frac{d(1-m)}{Y_{0}^{2}},
$$

where $Y_{0}$ is the smallest root of Eq. (18). In the special 
case $g_{2}=0, m=1\left(g_{3}=0\right), g_{1} \gg \sqrt{2}, Y_{0}=\sqrt{2} / g_{1} \ll 1$, we recover the result from [3]:

$$
j_{c}=\frac{Y_{0}}{2 \sqrt{2}}=\frac{1}{2 g_{1}}
$$

\section{NUMERICAL MODELLING}

We note that Eqs. (8)-(10), the boundary conditions at the boundaries $z= \pm L$, as well as the Weierstrass conditions at the points $z= \pm d / 2$, can be interpreted as a necessary extremum conditions for the free energy functional

$$
\begin{aligned}
F[R, \varphi] & =\int_{-L}^{L} \mathcal{L}\left(x, R, R^{\prime}, \varphi^{\prime}\right) d x \\
& +J[\varphi(-L)-\varphi(L)] .
\end{aligned}
$$

Here, the energy density $\mathcal{L}$ is given by

$$
\mathcal{L}=\frac{1}{2}\left\{\begin{array}{c}
R^{\prime 2}+R^{2} \varphi^{\prime 2}-R^{2}+\frac{1}{2} R^{4}, \quad z \notin\left(-\frac{d}{2}, \frac{d}{2}\right) \\
\frac{1}{m}\left(R^{\prime 2}+R^{2} \varphi^{\prime 2}+\alpha R^{2}+\frac{\beta}{2} R^{4}\right), \quad z \in\left(-\frac{d}{2}, \frac{d}{2}\right) .
\end{array}\right.
$$

The Generalized Continuous Analogue of Newton's Method (see the survey by Puzynin et al. [8]) for solving the nonlinear differential equations (8) and (9) on the finite interval $z \in(-L, L)$ with zero Neumann conditions at the boundaries $z= \pm L$ and appropriate conditions at $z= \pm d / 2$, is applied. At each iteration the corresponding linear boundary value problem is solved numerically using the finite elements method on a nonuniform grid, condensed to the boundaries $z= \pm d / 2$ of the layer. Then the corresponding phase difference $\varphi(z)$ is calculated by means of the integral (10).

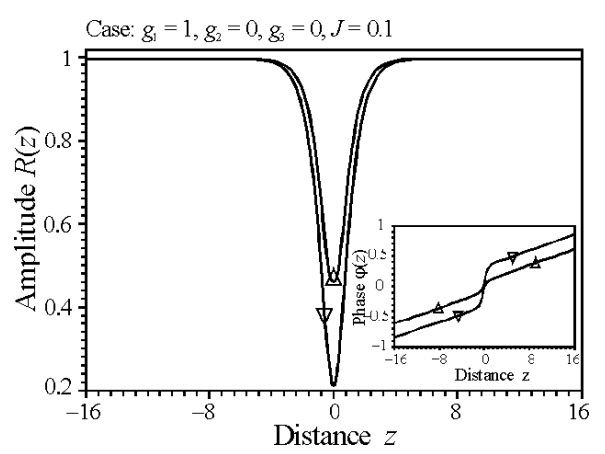

Fig. 1. The basic two solutions for $J=0.1, g_{1}=1, g_{2}=0$ and $g_{3}=0$.

All numerical results from now on were obtained for $L=16$ and width of the layer $d=0.2$. The main two solutions $R(z, J)$ we found numerically are demonstrated in Fig. 1 (in this case $J=0.1, g_{1}=1, g_{2}=$ $0, g_{3}=0$ ). The first solution (marked by $\triangle$, full energy $F \approx-2.46)$ exists in the interval $J \in\left[-j_{c}, j_{c}\right]$, whereas for the second one (marked by $\nabla, F \approx-2.39$ ) we have
$J \in\left(0,-j_{c}\right] \cup\left[j_{c}, 0\right)$. The first solution originates from the "uniform" solution $R(z)=1, \varphi(z)=0$, existing in the case when $g_{1}=0, g_{2}=0, g_{3}=0$, and $J=0$. The process of generation of the order parameter's amplitude $R(z)$, when $g_{1}$ increases, is shown in Fig. 2.

It is necessary to note, that except the above mentioned basic solutions there exist a number of highenergy solutions, some of which are demonstrated in Fig. 3. These solutions can be considered as a chain of $N>1$ solitons and the corresponding energy increase when the number $N$ increases. As can be seen, the solutions of such a kind can be interpreted as a nonlinear interaction between one of the basic solitons, localized at the point $z=0$, and even number symmetrically situated solitons on its left and right.

The graphics displayed in Fig. 4 correspond to the $J(\Delta \varphi)$ curves for four different values of $g_{1}\left(g_{1}=0, g_{1}=\right.$ $1, g_{1}=5$, and $\left.g_{1}=10\right)$ at $g_{2}=0$ and $g_{3}=0$.

If the quantity $g_{1}=0$ (the corresponding curve is marked by $\square$ ) the maximum is achieved at $j_{\text {dep }}=$ $2 / 3 \sqrt{3} \approx 0.385$ (the depairing current density in an uniform superconductor). For large values of $g_{1}$ we found results close to the ideal Josephson relation (1), which will be analyzed more strictly below. We note that the numerical results displayed in Fig. 4 are in a good agreement with Fig. 2 in [3]. For each curve in this figure we denote $j_{c}=\max J(\Delta \varphi)$ when $\Delta \varphi / \pi \in(-1,1)$.

The dependence of the free energy $F(J)$ on the current density $J$ for these two solutions is represented graphically in Fig. 5 for different values of $g_{1}$. This is a typical bifurcation diagram: at the points $B$ where $J=j_{c}$, the two branches, which correspond to the solutions with different energies, coalesce and acquire a common cusp.

For completeness the dependence of full energy $F(\Delta \varphi / \pi)$ on normalized phase difference is demonstrated in Fig. 6 for different values of $g_{1}, g_{2}=0$, and 
$g_{3}=0$. The minima $B$ of these curves correspond to the critical current density $\pm j_{c}$ (see Fig. 4).

Figs. 7 and 8 represent the influence of the parameters $g_{2}$ and $g_{3}$, respectively, on the $J(\Delta \varphi)$ curve. A comparison between Fig. 7 and Fig. 4 at $g_{1}=1$ clearly indicates the influence of the parameter $g_{2}$ on the current density $J$ - with the enlargement of $\left|g_{2}\right|$ we have more pronounced Josephson behaviour of the curve $J(\Delta \varphi)$.

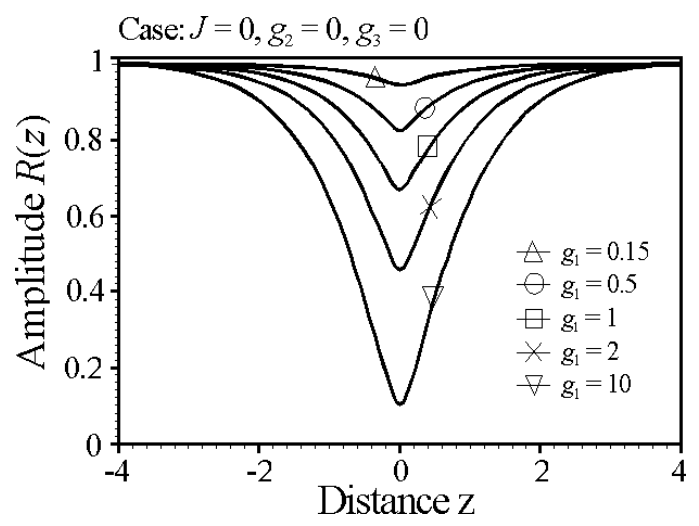

Fig. 2. The amplitude $R(z)$ increase along with the parameter $g_{1}$.

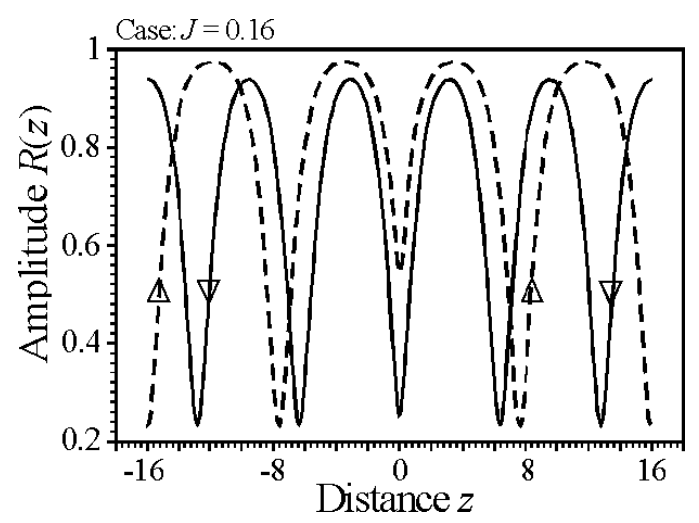

Fig. 3. Chains of high-energy solutions: $\Delta-N=3, F \approx-0.68 ; \nabla-N=5, F \approx-0.07$

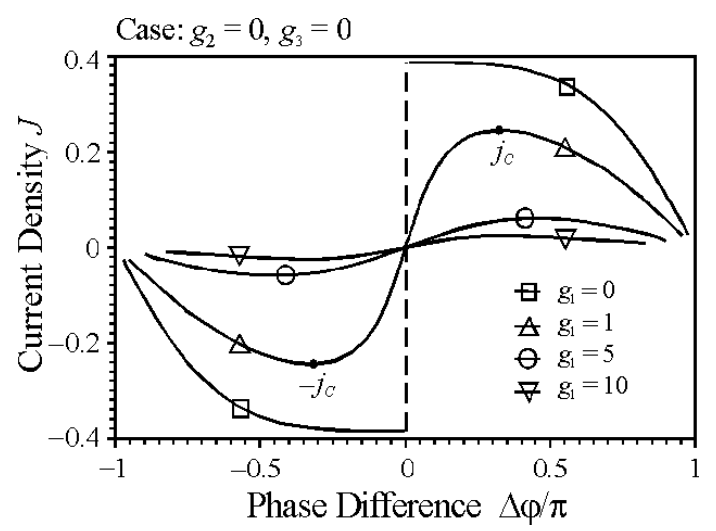

Fig. 4. Some typical curves $J(\Delta \varphi)$ for $g_{2}=0$ and $g_{3}=0$.

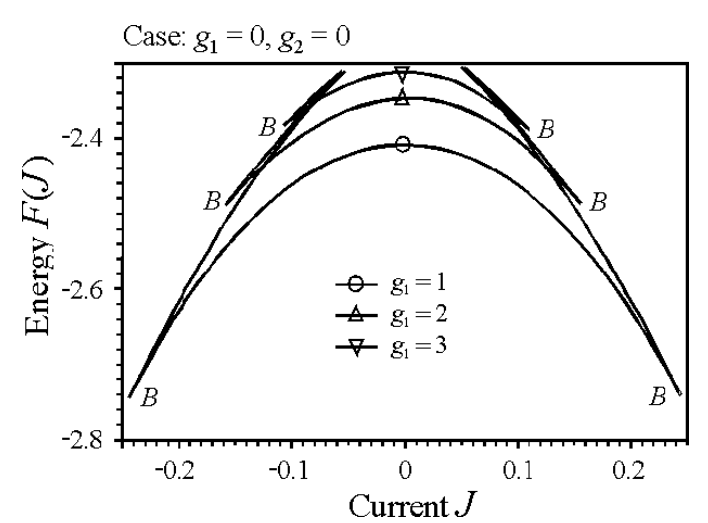

Fig. 5. The critical current $j_{c}$ corresponds to a bifurcation of basic solutions.

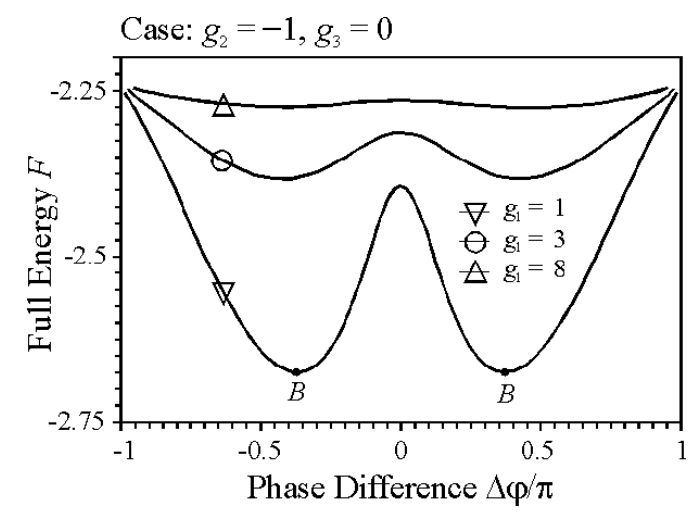

Fig. 6. The critical current $j_{c}$ corresponds to the minimum of full energy.

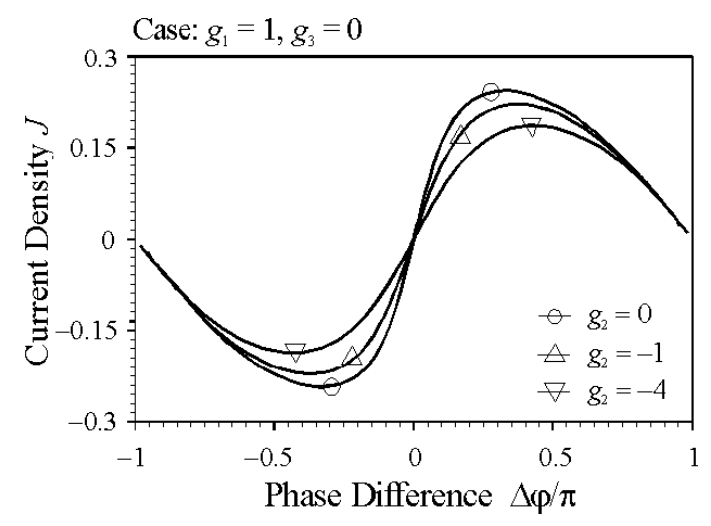

Fig. 7. The influence of the parameter $g_{2}$.

A comparison between Fig. 8 and Fig. 4 also for $g_{1}=1$ shows that the variation of the parameter $g_{3}$ between 0 and -3.5 leads to a significant reduction of the maximum current density (approximately twofold).

These quantitative conclusions can be coupled with the Fourier decomposition of $J(\Delta \varphi)$ curves as given by 
Eq. (2). We restrict ourselves only to the analysis of the ratio $a_{2} / a_{1}$ of the first two Fourier coefficients. When $a_{2} / a_{1} \ll 1$ we have approximately a pronounced Josephson behaviour $J \simeq j_{c} \sin \Delta \varphi$ since $j_{s} \approx a_{1}$.

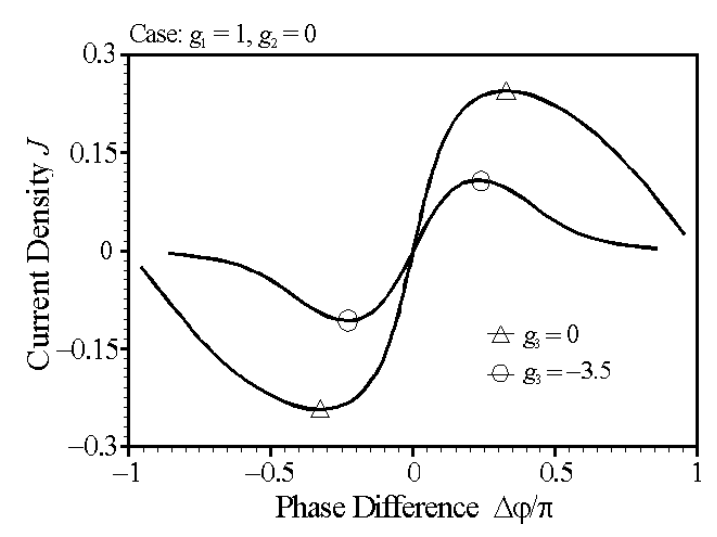

Fig. 8. The influence of the parameter $g_{3}$.

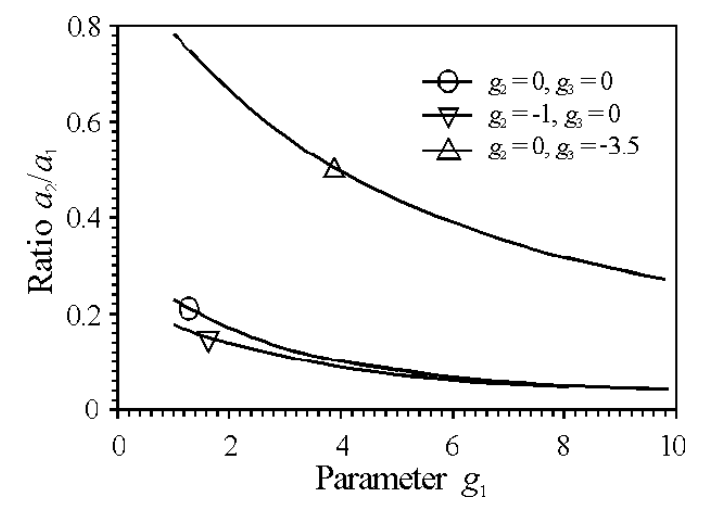

Fig. 9. The ratio $a_{2} / a_{1}$ of the first two Fourier coefficients as a function of parameter $g_{1}$.

The ratio $a_{2} / a_{1}$ as a function of the parameter $g_{1}$ is shown in Fig. 9. It is seen that for large enough values of the parameter $g_{1}\left(g_{1}>8\right.$, see [3]) when the parameter $g_{3}=0$, the coefficient $a_{2}$ is less than $5 \%$ of $a_{1}$. On the contrary, for small values of $g_{1}$ we have a substantial weight of higher harmonics (for example, if $g_{1}=1$ then the ratio $a_{2} / a_{1} \approx 0.23$ ).

As can be expected (see the curve marked by $\nabla$ ), the influence of the change of the parameter $g_{2}$ on the Fourier coefficients is essential for small enough values only of the parameter $g_{1}$ (for example, if $g_{1}=1, g_{2}=-1$, and $g_{3}=0$, then $\left.a_{2} / a_{1} \approx 0.17\right)$. On the other hand, taking into account the coefficient $g_{3}<0$ (the ratio $m>1$ ) leads to a significant increase of the second term in Eq. (2) even at great values of the parameter $g_{1}$ (the corresponding curve $a_{2} / a_{1}$ is marked by $\triangle$ ). This fact can be considered as an argument for applying the double $\mathrm{SG}$ equation [9] to some SNS structures.

Fig. 10 shows the comparison between numerically obtained and theoretically calculated curves $j_{c}\left(g_{1}\right)$ by means of formula (20) for $g_{2}=0$ and $g_{3}=0$. We emphasize the agreement between the theoretical and numerically obtained relations.

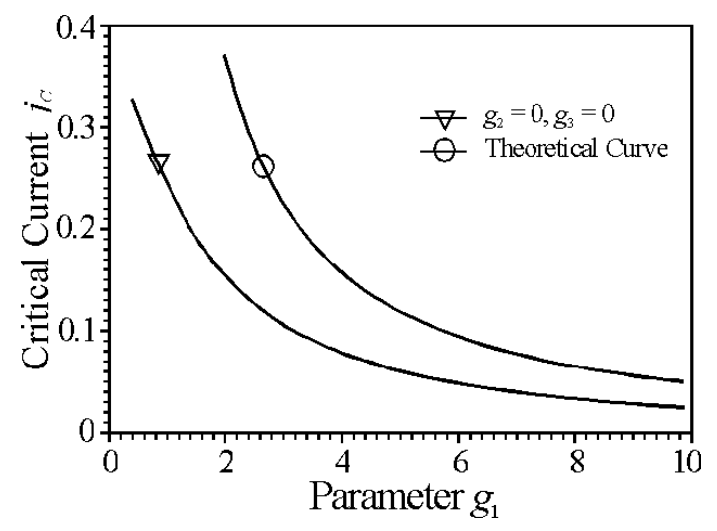

Fig. 10. The critical current $j_{c}$ as a function of the parameter $g_{1}$.

\section{CONCLUDING REMARKS}

In the present paper we show that by taking into account different nonlinear terms in the normal and superconducting regions in SNS sandwich, many harmonics exist and the dependence $J(\Delta \varphi)$ of the current as a function of the phase offset is not sinusoidal. The usually accepted sinusoidal dependence $J=j_{c} \sin (\Delta \phi)$ is justified only for a restricted domain of values of the parameters $d, m_{n} / m_{s}, a_{n} /\left|a_{s}\right|$, and $b_{n} / b_{s}$.

We prove numerically that the essential deviation from sinusoidal relation is caused by the possible anisotropy of masses $\left(m_{n} / m_{s} \neq 1\right)$. The numerical investigation indicates nontrivial specific modifications of the $J(\Delta \varphi)$ curve by the introduced new parameters $g_{2}$ and $g_{3}$. When the anisotropy of masses is absent $\left(m_{n} / m_{s}=1\right), b_{n}=b_{s}$ and the thickness $d$ is very small, we recover the results given in [3].
[1] K. K. Licharev, Rev. Mod. Phys. 51, 101 (1979).

[2] J. R. Waldram, Rep. Prog. Phys. 39, 751 (1976).

[3] F. Sols and J. Ferrer, Phys. Rev. B 49, 15913 (1994).

[4] S. J. Chapman, Q. Du, M. D. Gunzburger, Euro. J. Appl.
Math. 6, 97 (1995).

[5] K. H. Hoffman, L. Jiang, W. Yu, N. Zhu, Math. Methods Appl. Sci. 21, 59 (1998).

[6] Q. Du, J. Remski, Euro. J. Appl. Math. 10, 1 (1999). 
[7] M. Nishida, N. Hatakenaka, S. Kurihara, preprint condmat/0108368 (2001).

[8] I. V. Puzynin, I. V. Amirkhanov, E. V. Zemlyanaya, V. N. Pervushin, T. P. Puzynina, T. A. Strizh,

V. D. Lakhno, in Physics of Elementary Particles and
Atomic Nuclei 30, No 1, (1999) 97, AIP.

[9] R. K. Bullough, P. J. Caudrey, H. M. Gibbs, in: Solitons, edited by R. K. Bullough, P. J. Caudrey, (Springer-Verlag, New York, 1980).

\title{
ДОСЛІДЖЕННЯ МОДИФІКОВАНОГО РІВНЯННЯ ГІНЗБУРГА-ЛАНДАУ ДЛЯ ДЖОЗЕФСОНІВСЬКОГО КОНТАКТУ
}

\author{
Т. Л. Бояджев ${ }^{1}$, 3. Д. Генчев ${ }^{2}$ \\ ${ }^{1}$ Факультет математики і комп'ютерних наук, \\ Університет Софї "Сб. Кл. Отрідскі", \\ $B G-1164$, Софiл, Болеаріл \\ E-mail: todorlb@fmi.uni-sofia.bg \\ ${ }^{2}$ Iнститут електроніки, Болгарсъка академіл наук, \\ $B G-1784$, Софіл, Болгаріл \\ E-mail: zgenchev@ie.bas.bg
}

Формалізм Гінзбурга-Ландау (ГЛ), дає простий метод для дослідження глобальних властивостей неоднорідних надпровідних структур. У цій роботі досліджено клас структур надпровідна звичайна надпровідна (НЗН) (сендвічі) з плоскими гранипями на основі рівнянь типу модифікованого рівняння Гінзбурга-Ландау (ЦЛ). Відповідну нелінійну задачу граничного значення амплітуди параметра порядку розв'язано чисельно. Показано, що для фіксованих значень феноменологічних коефіпієнтів НЗН структури існують різні розв'язки з різними енергіями і власною різнищею фаз. Також аналітично отримано два основні розв'язки з мінімальною енергією для нескінченного сендвіча. Отримано залежність густини струму від зсуву фази. Завдяки існуванню різних нелінійних доданків у звичайній та надпровідній ділянках пі залежності не $є$ синусоїдальними. Також зроблено фур'є-розклад кривої залежности густини струму ві,д зсуву фази для того, щоб оџнити вплив феноменологічних коефіпієнтів на форму пієї кривої. 